\title{
Analysis of Salt Stratification Modeling Influences over Reverse Time Migration Considering and not Considering Least-Squares Schemes
}

\author{
Alexandre Maul (1,2)*; Roberto Dias (1); Leonardo Teixeira (1,2); Filipe Borges (1); André Bulcão (1); \\ Bruno Pereira-Dias (2); María González (3); Cleverson Guizan (2) \& Marco Cetale (2)
}

(1) Petrobras S.A., Rio de Janeiro, RJ, Brazil;

(2) Universidade Federal Fluminense, Niterói, RJ, Brazil;

(3) Emerson Automation Solutions, Rio de Janeiro, RJ, Brazil.

\section{Copyright 2021, SBGf - Sociedade Brasileira de Geofísica}

This paper was prepared for presentation during the $17^{\text {th }}$ International Congress of the Brazilian Geophysical Society held in Rio de Janeiro, Brazil, 16-19 August 2021.

Contents of this paper were reviewed by the Technical Committee of the $17^{\text {th }}$ International Congress of the Brazilian Geophysical Society and do not necessarily represent any position of the SBGf, its officers or members. Electronic reproduction or storage of any part of this paper for commercial purposes without the written consent of the Brazilian Geophysical Society is prohibited.

\begin{abstract}
Seismic amplitude attribute is key aspect for reservoir characterization studies such as when using a trend information to populate the reservoir grids with properties. Therefore, it also poses some challenges due to the intrinsic uncertainties of the seismic experiment, especially under complex geological environments as we can see when dealing with the pre-salt reservoirs in the Santos Basin, offshore Brazil. The combination of detailed and reliable velocity models for the salt section and a suitable algorithm for seismic imaging seem to be the best way to achieve good results for both data quality (amplitude) and depth positioning. However, even getting this blend regarding the state-of-the-art of detailed velocity model and depth-migration algorithms, another composition with an amplitude-compensation scheme to correct the effects caused by uneven seismic illumination is mandatory. In this work, we present the benefits of using a reliable velocity model - with stratification of the evaporitic section - and reverse time migration, as well as considering or not the application of least-squares schemes. We perform qualitative and quantitative analysis of the results in different imaging scenarios, comparing key geological features and horizons. The results suggest that salt stratification has a significant impact on the depth positioning of the seismic events, while least-squares migration plays a more significant role in recovering the seismic amplitude.
\end{abstract}

\section{Introduction}

Remarkably seismic imaging in complex geological environments is challenging (Zhang and Sun, 2009; Huang et al., 2010; Jones and Davison, 2014). For the pre-salt reservoirs in the Santos Basin, offshore Brazil, which are great examples of such conditions, high-quality seismic images play a crucial role in exploration and development of the fields (Cooke et al., 2012). For the case of geological complexity, e.g. near-to-prominent salt bodies, there are significant improvements achieved when considering full waveform inversion (FWI) to update the velocity model prior to migration procedures (Tarantola, 1984; Zhang and Wang, 2009; Kang et al., 2019). On the other hand, reverse-time migration (RTM) is currently the most suitable algorithm for that kind of complex environments, and it requires a refined velocity model, which captures the overburden complexity (Jones and Davison, 2014; Kang et al., 2019; Shadrina et al., 2020). If such detailed velocity model, especially regarding the salt section, is not available, RTM images can deliver suboptimal amplitude response (Guo and Fagin, 2002; Zdraveva et al., 2011). Thus, in this challenging scenario FWI has successfully been used updating the velocity model prior any application of imaging algorithms, especially as an optimization problem such as in the least-squares schemes technique (Vigh et al., 2019).

Johann et al. (2013) state that a significant portion of the seismic used to characterize the reservoirs in the Santos Basin had considered tomography-derived velocity models for the evaporitic section for the migration processes. They comment that the initials models for these tomographic inversion usually take salt flooding approach (around $4,500 \mathrm{~m} / \mathrm{s}$ ), which mainly reflects the velocity response of halite-rich formation, once this is the more abundant mineral in this salty section (around $80 \%$ of occurrence). However, Ji et al. (2011) suggest the use of relative acoustic impedance as a proxy for the existing heterogeneities inside the salt section, in order to improve the results of depth migration algorithms in the same basin.

Currently the improvement of salt velocity estimations in the Santos Basin is an active field of research. Maul et al. (2015) propose a novel method to quantify and incorporate the well-observed existing stratification in the evaporitic sections, reflecting the salt-cycle of precipitation. Later, the inclusion of stratifications in the salt section building more geological velocity prove to be valuable for seismic processing (Gobatto et al., 2016; 
Falcão, 2017; Maul, 2020), even without well-log information to calibrate the models.

Despite playing an important role in seismic imaging of subsalt reservoirs, the inclusion of salt stratification is not enough to solve all the imaging issues, because of the unbalanced amplitude below complex salt bodies which may not correctly represent the elastic contrasts in subsurface. Therefore, least-squares schemes seem to be as a cutting-edge tool helping to compensate any poorly illuminated seismic amplitude (Schuster, 1993; Nemeth et al., 1999), and remarkably improving the seismic quality below the salt section, especially in the Santos Basin such as demonstrated in Pereira-Dias et al. (2017); Pereira-Dias et al. (2018); Shadrina et al. (2020). $\mathrm{Hu}$ et al. (2016) also presented enhancement in the resolution when considering least-squares schemes associated with the Gaussian Bean Migration (LSGBM), which confirms the improvements in resolution when adopting least-squares schemes with migration algorithms.

The main goal of the least-squares schemes is to recover the best reflectivity model according to a functional that measure the misfit between the observed and simulated dataset assuming a propagation model based on the Born's approximation. It mitigates distortions in poorly illuminated areas and extending the data bandwidth and, hence, its resolution. Nevertheless, this application is particularly sensitive to errors in the velocity model, which reinforces the necessity for accurate and detailed model building schemes in order to capture the wave propagation and its illumination effects (Luo and Hale, 2014, Shadrina et al., 2020).

In this paper, we explore the results of the combination of improved velocity models - including the stratification insertion in the salt section - and reverse-time migration, adopting or not the least-squares schemes. We performed qualitative (visual inspection of the images) and quantitative comparisons (frequency content of the data, and depth positioning and amplitude in some key horizons) covering different imaging scenarios, allowing us the evaluation and confirmation of the benefits of each technique, individually and associated. The emphasis is the benefit in using a detailed velocity model for the salt section, in this case adopting the methodology of stratification insertion, and the least-squares scheme using reverse-time migration in order to have the most reliable results taking all the advantages of these three methodologies together.

\section{Method}

In order to generate the study scenarios, we built a synthetic geological model, having a field data acquired in the Santos Basin as the origin. Our study focuses on the benefits of the usage of the stratification insertion when constructing the salt velocity model for the migration purposes. Here, we assume no anisotropy consideration for any scenarios. Thus, the difference in the subsalt seismic images of this study is exclusively due to the inclusion of the stratification into the velocity model and the usage or not of least-squares schemes. The preparation of the property model for the forward seismic modeling are described in details by Maul (2020) and the workflow followed the steps:

a) Selection of a 2D line from the 3D seismic dataset, containing amplitude and impedance (the impedance was delivered from a impedance inversion using the same amplitude data and all the available wells);

b) Insertion of three horizons (R1, R2 and R3) creating pre-salt geological features horizons into the 2D line (item "a"), generating contrasts of elastic properties (velocity and density), which are used as control points/features for comparison of depth and amplitude through the modeled scenarios;

c) Forward modeling of streamer seismic survey from the item "b". This is the synthetic data that we migrate using all the built velocity models explained in the following sequence;

d) Least-squares reverse time migration of the synthetic amplitude data using the reference velocity model, as described in step $b$, resulting in our reference model, from now called as LSRTM(REF);

e) Reverse-time migration of the synthetic amplitude data using the tomography-derived velocity model from the processing of the field data, resulting in the RTM(TOMO) image;

f) Use of RTM(TOMO) as input for a new impedance inversion. The low-frequency model was built using the five pseudo-well logs containing only the log information from the salt section. The result of the impedance inversion is combined with empirical correlations to convert the acoustic impedance into velocity model (Teixeira and Lupinacci, 2019; Maul, 2020). This is the impedance-derived velocity model;

g) Another reverse-time migration of the synthetic amplitude data using the impedance-derived velocity model , generating RTM(INV) image;

h) Least-squares reverse-time migration of the synthetic amplitude data using the tomographyderived velocity and the impedance-derived velocity model allowing us to obtain respectively LSRTM(TOMO) and LSRTM(INV) images;

i) Evaluation the quality and the quantification of the differences between LSRTM(REF) and the other four results: RTM(TOMO), RTM(INV), LSRTM(TOMO) and LSRTM(INV).

In this work, we used as a source a Ricker wavelet with a cutoff frequency of $45 \mathrm{~Hz}$, due to computational limitations. We used the reference property model (item "b") to simulate the seismic acquisition through a forward finite-difference modeling program employing the two-way acoustic wave equation, with non-reflecting boundary condition at the top. We modeled a narrow streamer seismic acquisition using the acoustic two-way wave equation with constant density. The synthetic dataset was reverse-time migrated with a slightly smoother velocity model version from our geological velocity model (a Gaussian filter was used in the slowness to avoid backscattering artifacts as defended by Loewenthal et al. (1987). Complementing our routine, we performed a 
least-squares scheme, which generates our "perfect solution" regarding seismic migration. Figure 1 exemplifies the model we considered (including the geological features and the horizons we are interested in be evaluating) as well as our so-called "perfect solution" regarding seismic migration, i.e, a least-squares reverse time migration having the input velocity model the same of each we generated our synthetic data.

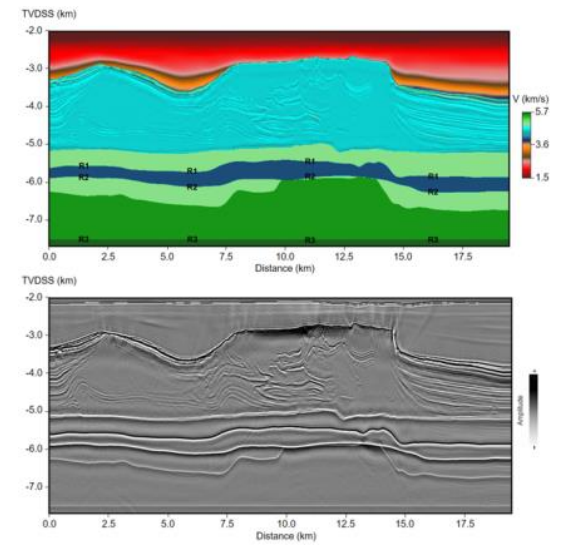

Figure 1 - Geological velocity model, including the features and the positioning of the three reference horizons used for quantitative used to analyze the results (A); and the obtained migrated (least-squares reverse time migration) using the velocity property model as presented in " $A$ " (B) - LSRTM(REF).

\section{Results and Analysis}

The synthetic data also underwent reverse-time migration with two other velocity models: the velocity obtained from pre-stack tomography on the field data, resulting in the image called RTM(TOMO); and a new impedance inversion model, built using the previous migrated data five pseudo-wells we took from the geological velocity model (salt portion), called RTM(INV). Over these two new migrated image we performed the application of least-squares schemes, generating LSRTM(TOMO) and LSRTM(INV).

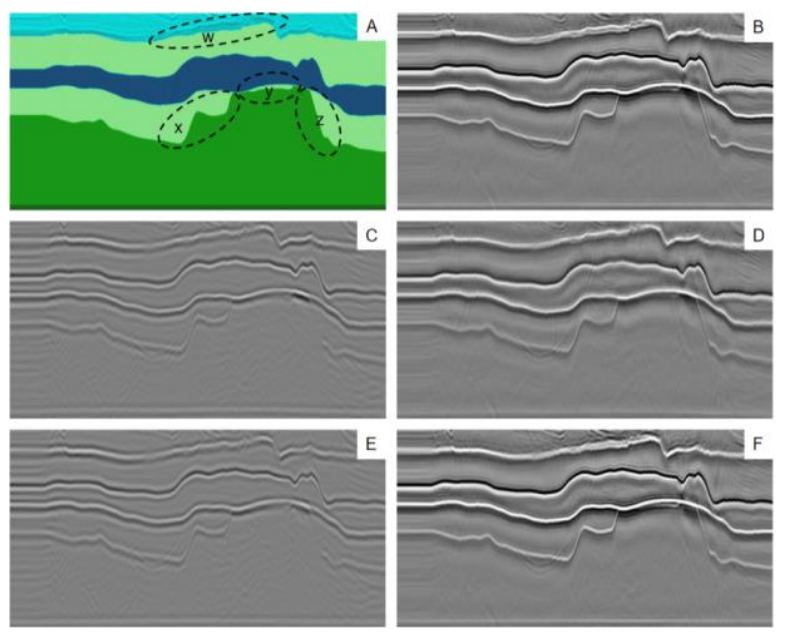

Figure 2 - Panels for visual inspection of data quality. (A) Geological model used for forward finite-difference modeling. Ellipses " $\boldsymbol{w}, \boldsymbol{x}, \boldsymbol{y}$ and $\boldsymbol{z}$ " highlights portions to analyze. (B) LSRTM(REF). (C) RTM(TOMO). RTM(INV). (E) LSRTM(TOMO). (F) LSRTM(INV).

Figure 2 summarizes all the images we generated. Note that in (A) we pointed four geological features (ellipses) in order to assess the quality analysis of the generated seismic image. In ellipse "w" we have 2 horizons at the base of salt, which we would expect to resolve in the seismic migrated image; ellipse " $x$ " shows a continuous horizon with significant depth variations and steep dip; ellipse " $y$ " displays a thin bed below a structurally complex overburden and ellipse " $\mathbf{z}$ ", another strong dip. The reference scenario (B) properly imaged all four features. However, the resulting image using tomographyderived velocity $(\mathbf{C})$ fails to reproduce any of the four highlighted features. This allow us to state that RTM algorithm, despite using the tomography-derived velocity model, is unable to recover the minute details that we are interested in such as increase of events resolution, amplitude response confidence. Application of leastsquares schemes to this image results in (D), which shows significant improvement in the continuity of the horizons over the initial result, despite not reproducing the main highlighted features. By improving the tomographyderived velocity model with the insertion of salt stratification, we can re-migrate the synthetic amplitude data obtaining the image shown in (E). The result is in fact very similar to the one in $(\mathbf{C})$, showing what seems to be little to no improvement. However, when performing least-squares schemes using the impedance-derived velocity model, the result $(\mathbf{F})$ shows the same continuity gains as in figure (D), but now with increased resolution similar to the resolution found in (B).

This does not come as a surprise to us once the quality of seismic images is strongly dependent on the velocity models used for the migration (Huang et al., 2017), particularly in complex areas such as salt flanks and subsalt zones (Zhang and Sun, 2009; Jones and Davison, 2014). Therefore, building geologically constrained velocity models, accounting for salt stratification, is imperative when imaging the pre-salt targets in the Santos Basin. The combination of a suitable migration algorithm (RTM) with least-squares schemes allows for further enhancement of the final image.

As one of the major goal of least-squares schemes is to recover the true reflectivity of the medium, the outcome of the process usually has a wider power spectrum, when compared with standard migration algorithms. As we built the seismic images in depth domain, to investigate the power spectrum, we converted the migrated images to the time domain, using the velocities of the respective migration models. The curves in figure 3 give us the quantitative confirmation of the analysis performed in figure 2: the application of least-squares schemes leads to a visible broadening of the data bandwidth, even for the tomography-derived velocity model (orange versus purple lines). It is in total accordance with the analytical demonstration described by Pereira-Dias et al. (2017). The more-accurate velocity significantly improves the seismic bandwidth, achieving results very close to the modeled data (gray), and reinforces the interpretation that velocity of intrasalt stratification in least-squares schemes yields better results. 


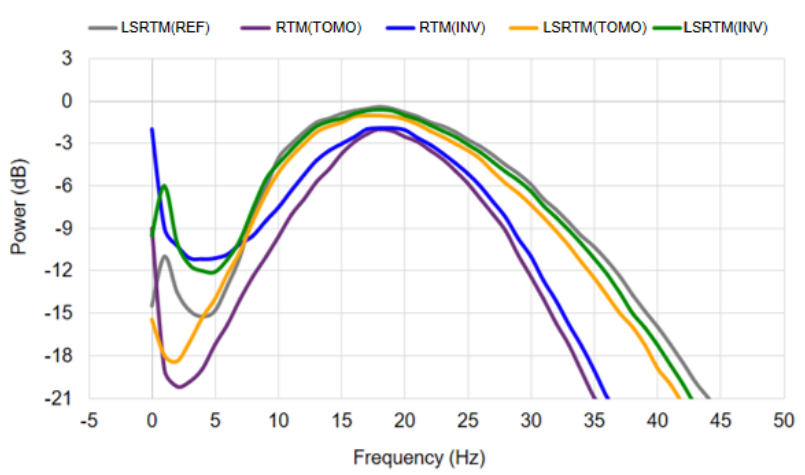

Figure 3 - Frequency content of the five built images, including our reference result LSRTM(REF).

Our first quantitative analysis presents the depth predictions delivered by the proposed combinations and its differences from LSRTM(REF) are shown in figure 4.
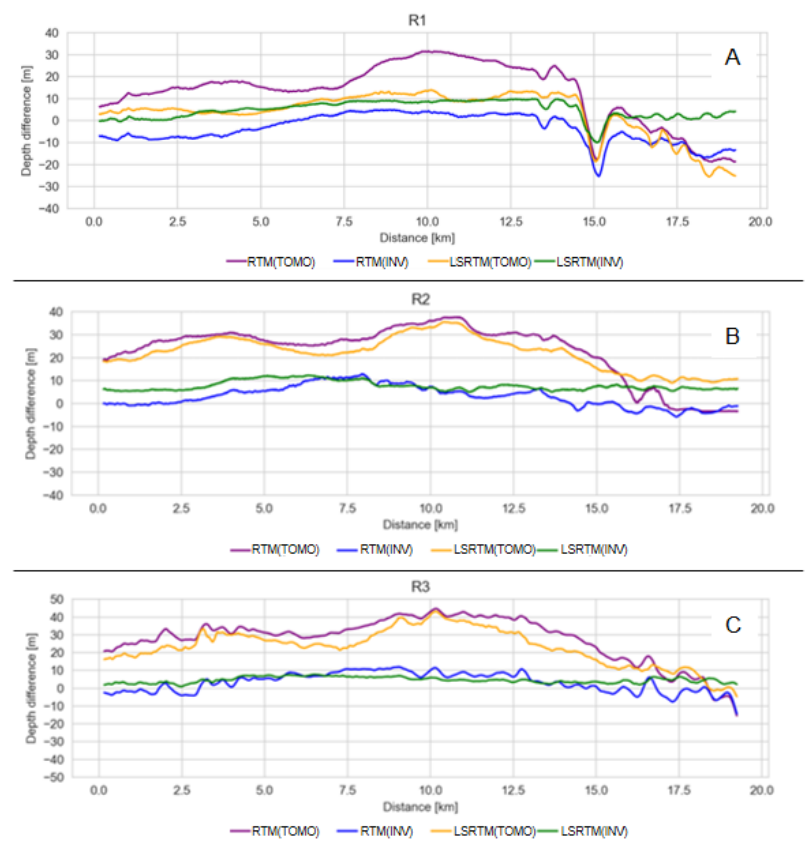

Figure 4 - Depth positioning differences at the three key horizons inserted into the model taking LSRTM(REF) as reference. $(\boldsymbol{A})$ reflects the differences of horizon "R1"; $(\boldsymbol{B})$ for horizon "R2" and (C) for horizon "R3".

Depth positioning is an important criterion when evaluating velocity models. In figure 4 we note that all the horizons that used the velocity model considering the salt stratification delivered accurate results and when performing the least-squares schemes are even better. Huang et al. (2010), after evaluating seismic images for the pre-salt prospects in the Santos Basin, attributed the poor depth predictability to the simplicity of the velocity models used for migration. Falcão (2017) presented results comparing Kirchhoff and RTM migration, using velocity models both with and without salt stratification, and emphasized the importance of considering the stratification in the salt velocity models. As we can see in our results, the stratified velocity model delivers good depth prediction even without the least-squares schemes, which reinforces that stratification is a good starting point for velocity model improvement. However, the depth accuracy becomes even better with the application of least-squares schemes, particularly for regions with poor illumination, such as near horizon R1.

Our second quantitative analysis presents the amplitude predictions delivered by the proposed combinations and its differences from LSRTM(REF) are shown in figure 5.
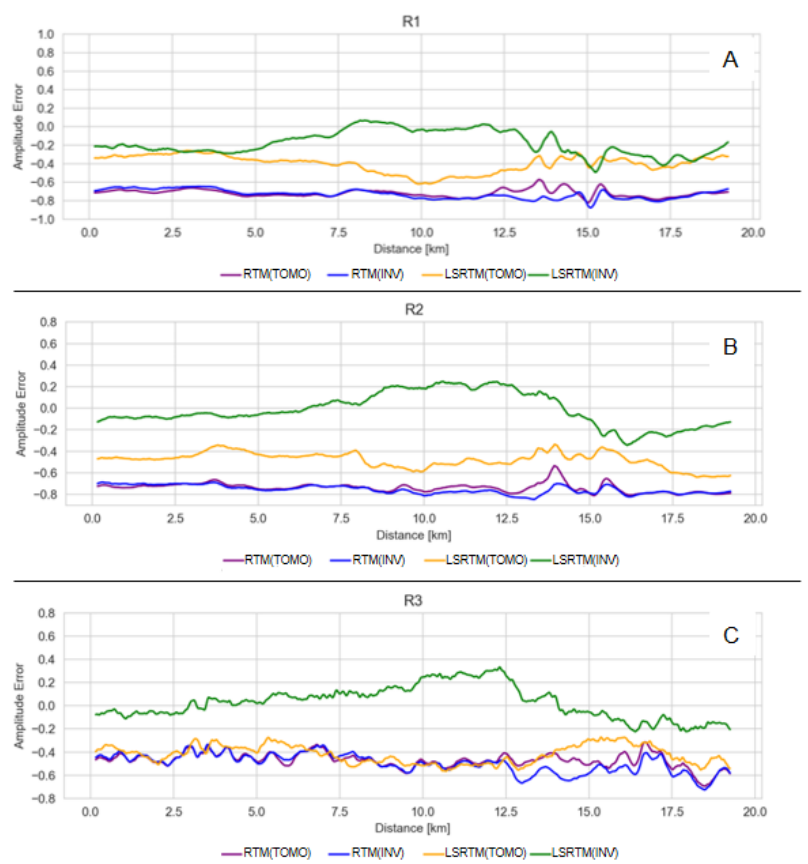

Figure 5 - Amplitude values difference at the three key horizons inserted into the model taking LSRTM(REF) as reference. (A) reflects the differences of horizon " $\boldsymbol{R 1}$ "; $(\boldsymbol{B})$ for horizon "R2" and (C) for horizon "R3".

Figure 5 shows the amplitude response at the same levels (R1, R2 and R3). It is clear that the combination of stratified velocity model and least-squares schemes delivers better results for all three horizons, when compared to the reference model. We can also note that when we add the detailed velocity model (with the stratification insertion) the results are even better. This last statement confirms that least-squares schemes in the velocity models associated with detailed velocity models has potential to deliver more accurate amplitude responses for the pre-salt targets in the Santos Basin, as pointed in some previous works (Ji et al., 2011; Wang et al., 2017; Fonseca et al., 2018; Dias et al., 2019; Shadrina et al., 2020).

\section{Conclusions}

Accounting for the heterogeneity of salt is pivotal for subsalt imaging and most of the wells drilled in the Santos Basin - pre-salt reservoirs has identified salt stratifications. Therefore, any velocity model built in the area should attempt to account for these heterogeneities. As well as, migration alone might not be enough to deliver true amplitude response. 
Thus, we proposed a non-standard seismic "migration" workflow combining the stratification insertion in the salt section, a reverse time-migration adopting least-squares schemes to ensure the best fidelity regarding the subsurface media.

Starting from a real dataset, we built elastic property models and simulated a seismic acquisition. The synthetic amplitude data was processed using different, attainable scenarios of velocity model building and imaging techniques. Migration of the synthetic amplitude data with a stratified salt velocity model was able to produce significant improvements in depth positioning, when compared to the use of a velocity obtained from tomography of field data. We observed an enhancement of depth predictions when using these stratified models with the least-squares schemes attached to reverse-time migration.

Despite the use of a more-accurate velocity model (the stratified one), uneven illumination caused by the acquisition geometry and complex overburden can create amplitude variations that are not in accordance with the contrast of elastic properties. Least-squares scheme is a handy tool to address this issue. As we can see through our results, the this scheme delivers good results, but the absence of a detailed velocity model (stratified) for the salt section leads to poor outcomes: several geological features were not properly imaged. Thus, the association of this least-squares scheme plus the stratified salt model plus reverse time-migration delivers the best results regarding the amplitude response.

Therefore, as anticipated, we defend that the combination of both methods is be the best choice for seismic imaging in this complex environment: the combined application of these techniques leads to improvements in the seismic images, with better depth positioning, resolution, event continuity and amplitude balance. The desirable consequence is that reliable seismic images bring forth a reduction of seismic uncertainties that leads to trust worthy rock property estimations and better identification of the internal architectures of the reservoirs. These are essential to improve reservoir characterization, strongly underpinning the decision-making in oil industry.

\section{Acknowledgments}

The authors would like express their gratitude to PETROBRAS for giving all the needed support and time to develop this research, as well as for allowing this publication, and to ANP to provide the data used for the tests.

\section{References}

Cooke, A., Le Diagon, F., Marco, R., Amazonas, D., Bunting, T., Moldoveanu, N., Klug, S. \& Mattos, E. 2012. Full-azimuth towed-streamer seismic: an exploration tool for pre-salt hydrocarbon exploration offshore Brazil. In: SEG International Exposition and 84th Annual Meeting. Las Vegas, NV, USA. 10.1190/segan2012-1076.1.
Dias, R., Fonseca, J., Bulcão, A., Pereira-Dias, B., Teixeira, L., Maul, A., Borges, F., 2019. Salt stratification and least-squares migration to improve pre-salt images: Santos Basin, Brazilian offshore example. In: Second EAGE/PESGB Workshop on Velocities). London, United Kingdom. 10.3997/2214-4609.201900051.

Falcão, L. 2017. O Sal Estratificado e sua Importância na Modelagem de Velocidades para Fins de Migração Sísmica. Master's Dissertation. UFF. Niterói, RJ, Brazil.

Fonseca J., Teixeira L., Maul A., Barros P., Boechat J. \& González M. 2018. Modelling Geological Layers into new Velocity Models for Seismic Migration Process: A Brazilian Pre-Salt Case. First EAGE/PESGB Workshop on Velocities. London, UK. doi: 10.3997/22144609.201800010.

Gobatto, F., Maul, A., Falcão, L., Teixeira, L., Boechat, J.B., González, M., González, G., 2016. Refining velocity model within the salt section in Santos Basin: an innovative workflow to include the existing stratification and its considerations. In: SEG International Exposition and 87th Annual Meeting. Dallas, TX, USA. 10.1190/segam2016-13685489.1.

Guo, N. and Fagin, S. 2002. Becoming effective velocity model builders and depth imagers, Part 2 - the basics of velocity-model building, examples and discussions. The Leading Edge, 1210-1216.

Hu, H., Liu Y., Zheng, Y. Liu, X. Lu, H. 2016, Leastsquares Gaussian beam migration. Geophysics, 81(3): S87-S100, (May-2016). 10.1190/geo2015-0328.1.

Huang, Y., Lin, D., Bai, B., Roby, S. \& Ricardez, C. 2010. Challenges in Pre-Salt Depth Imaging of the Deepwater Santos Basin, Brazil. The Leading Edge, 29(7): 820-825. doi: 10.1190/1.3462785.

Ji, S., Huang, T., Fu, K. \& Li, Z. 2011. Dirty Salt Velocity Inversion: The Road to a Clearer Subsalt Image. Geophysics, 76(5): WB169-W174. doi: 10.1190/GEO2010-0392.1.

Johann, P., Camargo, G.N., Penna, R.M., Dias, R.M., 2103. Challenges in seismic imaging and reservoir characterization of presalt oilfields in offshore Brazil. In: Offshore Technology Conference - OTC.

Jones, I. F. \& Davison I. 2014. Seismic Imaging in and around Salt Bodies. Interpretation, Vol. 2, No.4, SL1SL20. doi: 10.1190/INT-2014-0033.1.

Kang, W., M. Ortin, J. Monteiro, M. Fagundes, O. Zdraveva, D. Vigh, M. Shadrina, 2019, Improving pre-salt image with reflection full-waveform inversion in Santos Basin. In: First EAGE Workshop on Pre-Salt Reservoir: From Exploration to Production, 1-5. 10.3997/22144609.201982034.

Loewenthal, D., Stoffa, P.L., Faria, E.L., 1987. Suppressing the unwanted reflections of the full wave equation. Geophysics, 52 (7). 10.1190/1.1442352.

Luo, S., Hale, D., 2014. Least-squares migration in the presence of velocity errors. Geophysics, 79(4), (JulyAugust 2014). 10.1190/geo2013-0374.1. 
Maul, A. 2020. Caracterização Sísmica da Seção Evaporítica Salina e suas Aplicações nos Projetos de Exploração, Desenvolvimento e Produção de Hidrocarbonetos. Doctor's Thesis. UFF. Niterói, RJ, Brazil.

Maul, A., Jardim, F., Falcão, L. \& González, G. 2015. Observing Amplitude Uncertainties for a Pre-salt Reservoir using Illumination Study (Hit-maps). $77^{\text {th }}$ EAGE Conference and Exhibition. Expanded Abstracts. Madrid, Spain. doi: 10.3997/2214-4609.201412921.

Nemeth, T., Wu, C., Schuster, G.T., 1999. Least-squares migration of incomplete reflection data. Geophysics, 64, 208-221. 10.1190/1.1444517.

Pereira-Dias, B., Bulcão, A., Soares Filho, D.M., Santos, L.A., Dias, R.M., Loureiro, F.P., Duarte, F.S., 2017. Leastsquares migration in the image domain with sparsity constraints: an approach for super-resolution in depth imaging. In: 15th International Congress of the Brazilian Geophysical Society 2015. Rio de Janeiro, RJ, Brazil. Expanded Abstracts. 10.1190/sbgf2017-236.

Pereira-Dias, B., Guerra, C., Bulcão, A., Dias, R.M., 2018. Exploring inversion strategies in image domain leastsquares migration. In: First EAGE/SBGf Workshop on Least Squares Migration. Rio de Janeiro, RJ. Brazil. Expanded Abstracts. 10.3997/2214-4609.201803060.

Shadrina, M., Cavalca, M., Ortin, M., Pantoja, M., Medina, E., Leone, C., Fletcher, R., 2020. Prestack least-squares RTM on surface offset gathers for more reliable quantitative interpretation - Santos Basin case study. In: SEG International Exposition and 90th Annual Meeting. Houston, TX, USA (virtual event). 10.1190/segam20203427474.1 .

Schuster, G.T., 1993. Least-squares crosswell migration. In: SEG International Exposition and 63rd Annual Meeting. 10.1190/segam2012-1425.1.

Tarantola, A., 1984. Inversion of seismic reflection data in the acoustic approximation: Geophysics, 49, 1259-1266. 10.1190/1.1441754.

Teixeira, L., Lupinacci, W., 2019. Elastic properties of rock salt in the Santos Basin: relations and spatial predictions. Journal of Petroleum Science and Engineering, $\quad 180, \quad 215-230$. https://doi.org/10.1016/j.petrol.2019.05.024/22144609.201901460 .

Vigh, D, Jiao, K., Cheng, X., Sun, D., Glaccum, K, 2019. An offshore Gulf of Mexico case study applying fullwaveform inversion. Interpretation, 7(2): 1-35. https://doi.org/10.1190/INT-2018-0135.1.

Wang, P., Huang, H. Wang, M., 2017. Improved subsalt images with least-squares reserve-time migration. Interpretation, 5(3): SN25-SN32. 10.1190/INT-20160203.1.

Zdraveva, O., Woodward, M., Nichols, D., Osypov, K., 2011. Building anisotropic models for depth imaging: comparing different approaches. In: 12th International Congress of the Brazilian Geophysical Society. Expanded Abstracts. Rio de Janeiro, RJ, Brazil. Society of
Exploration Geophysicists and Brazilian Geophysical Society. 10.4043/22827-MS.

Zhang, Y., Sun, J., 2009. Practical issues of reverse time migration: true-amplitude gathers, noise removal and harmonic-source encoding. In: 70th EAGE Conference \& Exhibition. Rome, Italy. 10.3997/2214-4609.20147708.

Zhang, Y., Wang, D., 2009. Traveltime information-based wave-equation inversion: Geophysics, 74(6): WCC27WCC36. 10.1190/1.3243073. 